REVISTA ElectróniCA EdUCARE (EDUCARE EleCtroniC JOURNAL) EISSN: 1409-4258 VOL. 21(2) MAYO-AGOSTO, $2017:$ 1-25

[Cierre de edición 01 de mayo del 2017]

doi: http://dx.doi.org/10.15359/ree.21-2.12

URL: http://www.una.ac.cr/educare

CORREO: educare@una.cr

\title{
La ética en la investigación de posgrado
}

\author{
Ethics in Postgraduate Research
}

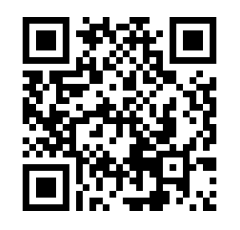

Recibido 30 de abril de 2016 • Corregido 21 de marzo de 2017 • Aceptado 18 de abril de 2017

\begin{abstract}
Resumen: En este artículo se exponen los resultados de una investigación exploratoria descriptiva con referentes empíricos. El objetivo consistió en documentar, sistematizar y valorar algunos rasgos de 15 tesis de posgrado en educación, con la intención de visibilizar algunas prácticas deshonestas recurrentes del alumnado y profesorado. Los programas de posgrado de donde se obtuvieron las tesis se encuentran localizados en el área metropolitana de la Ciudad de México. La selección de documentos se efectúo entre los meses de julio a noviembre de 2015. Se utilizaron dos escalas tipo Likert para valorar 2 dimensiones por separado: 1) contenido, en el cual se consideró originalidad, congruencia, dominio y pertinencia de autores, hallazgos y conclusiones relevantes y 2) presentación, en donde se examinó estilo, estructura lógica, ortografía y puntuación, citas, referencias y fuentes de información. A cada indicador se le otorgó un valor numérico y a este un rasgo valorativo: 5: excelente, 3: regular y 1: deficiente. Posteriormente se obtuvieron porcentajes. Los resultados indicaron que solo un tercio de las tesis pueden clasificarse como excelentes por la calidad del contenido y la impecable presentación; un porcentaje similar fueron trabajos que cumplieron con lo necesario, pero no se encontró el rigor y la originalidad de los primeros. Los trabajos restantes tuvieron serias deficiencias,
\end{abstract}

\footnotetext{
'Doctor en Pedagogía (UNAM). Miembro del Sistema Nacional de Investigadores (SIN) Nivel II (CONACYT). Forma parte de la planta académica del Programa de Posgrado en Pedagogía, UNAM. Investigador titular C del Instituto de Investigaciones sobre la Universidad y la Educación (UNAM). Ha formado parte de diversos comités evaluadores de proyectos y productos de investigación tanto en escuelas y facultades de la UNAM como en instancias externas, como CONACYT, ANUIES, CENEVAL, Secretaría de Relaciones Exteriores, COMIE. Ha sido coordinador de varios proyectos de investigación colectivos cuyas temáticas han sido la cultura ciudadana y la cultura política en estudiantes de educación superior: Imágenes sociales de la UNAM para el siglo XXI. Expectativas de actores de tres carreras; La construcción simbólica de las prácticas escolares; La UNAM vista por sus actores; La construcción social de la ciudadanía en agentes universitarios; La cultura política en estudiantes universitarios.

${ }^{2}$ Doctora en Pedagogía (UNAM). Miembro del Sistema Nacional de Investigadores (SIN) Nivel I (CONACYT). Forma parte de la planta académica del Programa de Posgrado en Pedagogía, FES Aragón (UNAM). Profesora investigadora en la Escuela Superior de Educación Física (SEP), en donde ha coordinado varios proyectos colectivos de investigación relacionados con la actividad física y salud en adolescentes de secundaria y con la enseñanza de la investigación en licenciatura. Ha publicado diversos artículos de investigación y participado en proyectos colectivos de investigación interinstitucional: Cultura ciudadana en instituciones de educación superior; Estudio de la ciudadanía en las escuelas normales; Estigma y discriminación en estudiantes normalistas, con instituciones como el IISUE, UNAM; FES Aragón; FES Zaragoza; FES Iztacala; DGENAM, SEP.
} 
doi: http://dx.doi.org/10.15359/ree.21-2.12

URL: http://www.una.ac.cr/educare

CORREO: educare@una.cr

tanto en el contenido como en la presentación formal del documento, sin alcanzar la mínima calidad que demanda una tesis de posgrado. El análisis de las investigaciones permitió visibilizar algunas de las prácticas deshonestas de las personas egresadas de diversos programas en educación y la falta de compromiso con su investigación. Los resultados acerca de la calidad de la investigación en diversos programas doctorales indican que hay poca responsabilidad en estudiantes, y personal tutor y revisor. Los resultados permiten que las autoridades de cada programa observen el problema y busquen las soluciones prácticas que permitan solucionarlo.

Palabras claves: Doctorado; ética; ethos de investigación; investigadores educativos; tesis.

\begin{abstract}
This article presents the results of an exploratory-descriptive research with empirical referents. The objective was to document, systematize and evaluate some features of 15 postgraduate theses in education, with the intention to make visible some recurring dishonest practices of students and teachers. The postgraduate programs from which the theses were obtained are located in the metropolitan area of Mexico City. The selection of documents was carried out between July and November 2015. Two Likert scales were used to assess two dimensions separately: 1) content, in which originality, congruence, dominance and relevance of authors, findings and relevant conclusions were considered; and 2) presentation, which examined style, logical structure, spelling and punctuation, citations, references and sources of information. Each indicator was assigned a numerical value and this one was given an evaluative characteristic: 5: excellent, 3: regular and 1: deficient. Subsequently, percentages were obtained. The results indicated that only a third of the theses could be classified as excellent for the quality of the content and the impeccable presentation; a similar percentage were works that fulfilled the necessary but didn't count with the rigor and originality of the first ones. The remaining papers had serious deficiencies, both in content and in the formal presentation of the document, without achieving the minimum quality demanded by a postgraduate thesis. The analysis of these investigations made it possible to visualize some of the dishonest practices of people graduating from various educational programs and the lack of commitment to their research. The results about the quality of the research in various doctoral programs indicate that there is little responsibility in students, personal tutor and the reviewer. The results allow the authorities of each program to observe the problem and seek practical solutions to solve it.
\end{abstract}

Keywords: doctoral studies; ethics; ethos research; educational researchers; thesis.

\title{
Introducción
}

Nuestro propósito en esta investigación fue analizar 15 tesis de posgrado para visibilizar algunas prácticas deshonestas en alumnado y tutorías de distintos programas de posgrado en educación de universidades nacionales. Nuestro supuesto considera que la tesis de grado sintetiza la formación ofrecida en el posgrado, en ella se forja aquello que el estudiantado logró incorporar durante su estancia en este espacio. El compromiso del estudiantado con su investigación, así como el apoyo proporcionado por la persona tutora y el comité tutor se plasman en los trabajos escritos. Y a la inversa, las prácticas que se desligan de este compromiso también se expresan en los documentos. Resultado: tesis excelentes, tesis regulares y tesis deficientes para los requerimientos de un posgrado.

Juan Manuel Piña-Osorio y Hilda Berenice Aguayo-Rousell

Los artículos de la Revista Electrónica Educare del Centro de Investigación y Docencia en Educación de la Universidad Nacional, Costa Rica, se comparten bajo términos de la Licencia Creative Commons: Reconocimiento, № Comercial, Sin Obra Derivada 3.0 Costa Rica. Las autorizaciones adicionales a las aquí delimitadas se pueden obtener en el correo: educare@una.cr 
En el primer apartado se examina la función del posgrado como instancia académica encargada de la formación en investigación. Es de esperar que en este espacio se le transmitan al estudiantado los conocimientos, metodologías y todo tipo de habilidades técnicas para realizar un trabajo de investigación, pero también las reglas morales de la disciplina (Merton, 2002) o ética profesional (Hirsh, 2003; Yurén, 2007). El ethos es un compromiso con la investigación, es realizarla de acuerdo con los parámetros de la disciplina. Sin embargo, se encuentra que el estudiantado y las personas tutoras son grupos heterogéneos, como también lo son las prácticas académicas. Por eso los trabajos de tesis son distintos, algunos muy cuidados y otros desatendidos.

En el siguiente apartado se analizan diferentes trabajos empíricos relacionados con prácticas no éticas o deshonestas académicamente. Las investigaciones son numerosas y reportan prácticas deshonestas en el posgrado. Las técnicas para profundizar en el problema han sido variadas, desde el empleo de cuestionarios con preguntas cerradas, hasta entrevistas realizadas a las personas participantes de un área académica. Esta revisión bibliográfica permite comprender que esta situación se ha registrado en mayor medida en los últimos años. Tal parece que, con la masificación de las redes, la información está a mano y es muy fácil cortarla y apropiarse de ella.

Posteriormente se presenta la estrategia metodológica seguida en esta investigación y el instrumento que se diseñó para evaluar las tesis, para buscar los elementos de contenido (originalidad, coherencia argumentativa, dominio de estudios, hallazgos y conclusiones relevantes) y presentación (estilo, estructura lógica, ortografía y puntuación, citas, referencias y bibliografía). No es nuestro interés señalar el programa de procedencia de cada tesis, decidimos mantenerlo en el anonimato.

En los dos últimos apartados se exponen los resultados de la indagación empírica y la discusión. En esta última se integran las formulaciones realizadas en otras investigaciones y los resultados del grupo de trabajo. Se analiza cómo las acciones y procedimientos de las personas profesionales del campo no siempre son éticas, situación corroborada al revisar los trabajos de investigación de posgrado. Conocer la calidad de las tesis en distintos programas doctorales permite profundizar en el compromiso del estudiantado y profesorado con sus actividades académicas. Develar esta situación posibilitará a los núcleos académicos comprometidos con su trabajo realizar los cambios pertinentes.

\section{El doctorado y la transmisión de un ethos}

Elestudiantadoque ingresa a un programa doctoral deberealizarnumerosas tareasacadémicas: leer, discutir y hacer reportes de lecturas, exponer su proyecto o un avance de investigación; elaborar ensayos, ponencias o artículos; estar en comunicación con la persona tutora y con quienes integran el comité, entre otras. Todo esto es un entrenamiento indispensable para elaborar la tesis de grado (Arnoux et al., 2004), para prepararse y convertirse en investigador o investigadora (Chavoya, 2001; Hamui, 2008; Moreno, 2013; Sánchez, 2000; Sánchez, Mireles y Jasso, 2000). 
doi: http://dx.doi.org/10.15359/ree.21-2.12

URL: http://www.una.ac.cr/educare

CORREO: educare@una.cr

Para un sector de estudiantes elaborar la tesis será un ejercicio indispensable en su formación. Comprenderá que esta actividad requiere dedicación, responsabilidad, revisión e interpretación de textos y conceptos, obtener e interpretar datos, expresar las ideas claramente, entre otras. Sin embargo, otro sector de estudiantes, por el contrario, tendrá dificultad para realizar la investigación o poco interés en esta tarea. La investigación es una profesión y la persona investigadora es portadora de un ethos. La elaboración de una tesis de grado bien hecha es un acto de ética profesional. Yurén (2007) apunta que un acto ético es un acto responsable y autónomo porque requiere la valoración de lo que se hace. El juicio valoral es el análisis y reflexión de las acciones futuras o pasadas. Es una deliberación prudencial para prever las posibles repercusiones de los actos. Sin este juicio se puede actuar irresponsablemente, porque el propósito se reduce a obtener beneficios personales o grupales. La autonomía permite discernir entre lo ético y lo no ético.

Para Merton (2002), el ethos de la ciencia es tanto el empleo de métodos como de imperativos institucionales, esto es, de normas técnicas y morales, que son para quienes participan en la ciencia, justas y orientadoras de las tareas. Integra códigos sociales, reglas y valores obligatorios para los científicos y las científicas. Contrariamente, quien no las cumple es porque no incorporó el ethos. Para Bolívar (2005), la ética profesional integra "el conjunto de principios morales y modos de actuar éticos en el ámbito profesional" (p. 96) y no son suficientes los conocimientos y habilidades de una profesión para desarrollar las tareas de la investigación.

El ethos proporciona estabilidad y conocimiento de las prácticas de un grupo científico; además, es la identidad con una disciplina o una profesión resultado de las interacciones entre colegas y docentes (Hamui, 2008). Es la incorporación de normas, principios y valores que orientan a quien investiga en las tareas del trabajo científico. Son los cimientos del compromiso ético y profesional en las personas doctorantes. Permite a quien se ha consagrado en la investigación, hacer bien su trabajo con base en los parámetros de la comunidad disciplinar, producto de una socialización científica. En la interacción se incorpora el "ejercicio cotidiano de la investigación" (Hamui, 2008, p. 98).

Sánchez (2000); Sánchez y Jasso (2002); Sánchez y Santamaría (2000) indagaron en numerosos programas de posgrado. Encontraron que en las aulas, pasillos, bibliotecas y en otros espacios, estudiantes y docentes discuten teorías, conceptos, metodologías, técnicas, investigaciones recientes, exámenes de grado, conferencias. El oficio de investigador o investigadora se logra cuando se poseen los conocimientos, las habilidades, además de una actitud ante lo que se hace (Sánchez, 2014). El ethos -compromiso ético- de la investigación, consiste en la incorporación de las reglas y conocimientos necesarios para investigar objetos educativos, implica tanto el dominio de un oficio como un compromiso con este. Chavoya (2001) concluyó que el trabajo de investigación está mediado por tres esferas: la disciplinar, o respeto a las normas; la institucional, o condiciones para realizar el trabajo de investigación; la 
del contexto, representado por las políticas educativas encarnadas en organismos evaluadores de las personas investigadoras, programas de posgrado y proyectos de investigación. La ética profesional consiste en hacer bien y responsablemente un trabajo, edificarlo de acuerdo con las normas establecidas dentro de un campo disciplinar (Chavoya, 2001; Hamui, 2008; Merton, 2002; Sánchez, 2014). La investigación educativa es una actividad académica. Se espera que las personas investigadoras actúen responsable y autónomamente, como parte indispensable de sus actos éticos (Serna, Roa, Martínez y Aguirre, 2014). Colina (2011) considera la ética profesional como una identificación con las normas y valores de la profesión, de manera que son una referencia existencial.

Sin embargo, en los programas de posgrado también se encuentran estudiantes con poco compromiso con el posgrado y muestran indolencia en las tareas académicas de los seminarios, como en la investigación de tesis. Moreno (2013) registró que el estudiantado de doctorado posee distintas trayectorias y diferentes motivos para estudiar. Pocas personas desean formarse como investigadores e investigadoras en una propuesta teórica y metodológica innovadora. Su deseo puede rebasar lo ofrecido dentro del programa doctoral, empero, lo buscan en otros espacios académicos, o bien, por cuenta propia a través de lecturas, conferencias, congresos. Otras personas, una mayoría, se forman solo con lo proporcionado por su programa. Resultado, diversidad de prácticas académicas y diversidad de tesis. Chavoya (2001) apunta algo más: el contexto de políticas de evaluación que ha provocado que el ethos de la investigación se interprete de diversas maneras incluso en un mismo programa. Sus repercusiones se observan en los procesos de indagación, en la certificación de programas de posgrado, en la incorporación de personal académico en el Sistema Nacional de Investigadores. Esto ha provocado en funcionarios, funcionarias y profesorado la gestación de acciones poco académicas o poco honestas, justificadas por la aprobación de las evaluaciones federales. Ahora es necesario que reporten las investigaciones en diferentes niveles educativos y en otros países.

\section{Prácticas no éticas visibles y no visibles de los actores educativos}

En el siguiente apartado se expondrá la revisión de bibliografía centrada en las prácticas no éticas o deshonestas en diferentes niveles educativos y en distintos países, situación que indica que se trata de un problema pertinente en el presente. Posteriormente, se analizarán distintas tesis doctorales que nos permitirán entender tanto el compromiso del estudiantado con su trabajo de investigación como la supervisión que su personal tutor y comité efectúan de este proceso de investigación.

La bibliografía especializada da cuenta de numerosas prácticas no éticas realizadas en los diferentes niveles educativos, las cuales caracterizan como deshonestas, fraude académico, vicios éticos, prácticas no éticas. Cierto tipo de prácticas se generan en todos los niveles educativos, mientras otras se efectúan básicamente en los programas de posgrado y en la investigación 
doi: http://dx.doi.org/10.15359/ree.21-2.12

URL: http://www.una.ac.cr/educare

CORREO: educare@una.cr

científica. Aluja y Birke (2004) realizan una clasificación de dos tipos de prácticas no éticas en la investigación científica. A unas denominan "éticamente inaceptables"(p. 98) ya otras "éticamente cuestionables" (p. 102). Quienes presentamos este estudio decidimos utilizar otra clasificación: prácticas no éticas visibles y no visibles. Las primeras se caracterizan porque son rechazadas por cualquier comunidad académica, son visibles para cualquiera; mientras las segundas, en numerosas ocasiones no son reconocidas como faltas, porque los usos y costumbres llegan a obnubilarlas. Se expondrán primero las prácticas visibles.

Por ejemplo, en España, Sureda y Comas (2009) indagaron las prácticas académicas deshonestas en estudiantes de universidades en el desarrollo de exámenes y trabajos académicos. Dentro de los primeros localizaron copiar en el examen, elaborar chuletas (notas para copiar en un examen), dejarse copiar, sustituir a un compañero en un examen, obtener previamente copia del examen, usar el celular, entre otros. Dentro de los segundos está usar y reproducir fragmentos o documentos completos sin proporcionar la fuente, tanto en documentos impresos como en red. Encontraron que 5 de cada 10 jóvenes han copiado a un compañero o se han auxiliado de una chuleta. Destacan que esto no es exclusivo del estudiantado español, porque otras investigaciones reconocen situaciones similares en estudiantes de EEUU, China, Rusia, Japón, Taiwán, Holanda, Moldavia, Croacia, Bulgaria y Serbia.

En otra investigación, Sureda, Comas y Morey (2009) analizaron el plagio del estudiantado desde la perspectiva del profesorado. Para ello diseñaron dos técnicas: un cuestionario que aplicaron a dos muestras de docentes y la formación de un grupo de discusión. Apuntan que las condiciones para esta práctica es la extensión de procesadores personales tanto en centros educativos como en el hogar, así como el uso masivo de internet y de numerosas redes que ofrecen documentos para cualquier temática. Mediante esta infraestructura, el estudiantado busca, encuentra y no pocas veces corta y pega información indispensable para tareas y trabajos académicos.

En Colombia, Díaz, González y Carmona (2010) encontraron que el fraude académico está difundido en los medios universitarios, lo cual repercute negativamente tanto en la formación disciplinar como en los valores profesionales indispensables para el mercado de trabajo. Las personas autoras llevaron a cabo una investigación con estudiantes en Cartagena, Colombia. Reconocen la heterogeneidad del estudiantado de la carrera de Odontología de la Universidad de Cartagena, porque provienen de zonas geográficas distintas, como también su situación religiosa, socioeconómica y cultural. Consideran que "las razones por las cuales cometen fraude académico están asociadas a situaciones personales derivadas o vinculadas a sus características demográficas particulares o a un contexto de permanencia ... con exigencias marcadas en lo académico" (p. 87). Este supuesto lo confirmaron con entrevistas estructuradas individuales, entrevistas en profundidad y un grupo focal. Encontraron que la mayoría de las personas entrevistadas señalaron haber cometido una falta deshonesta en algún momento; sin embargo, justificaron estas prácticas porque las consideraron indispensables para obtener 
una calificación aprobatoria. Por sus condiciones de vida, trabajan y estudian al mismo tiempo, además les interesa aprobar para que la familia se sienta orgullosa con un estudiante o una estudiante de universidad: El fin justifica los medios.

En México, Castillo-Méndez y Garibay-Pardo (2004) decidieron conocer la percepción del estudiantado acerca de los problemas éticos ante situaciones "poco éticas (en los vínculos) estudiante-maestro, estudiante-estudiante y estudiante autoridades"(p. 185). En un cuestionario preguntaron qué es la ética, los problemas éticos que inciden en la formación, la puntualidad y asistencia del profesorado, cumplimiento del programa, preocupación de este grupo por impulsar el desarrollo del alumnado, robo de información, venta de exámenes y calificaciones, discriminación de género o clase social en el aula, entre otras. El cuestionario se aplicó a 250 estudiantes de tres universidades nacionales. Los resultados arrojaron que un alto porcentaje de estudiantes expresó una opinión positiva de sus docentes; sin embargo, un porcentaje menor expresó lo contrario.

En otra investigación, Flores-Pérez (2004) aplicó un cuestionario a 189 estudiantes de especialidad, maestría y doctorado de instituciones públicas y privadas de México. Preguntó: ¿Qué entiende usted por ética? ¿La formación en su posgrado es ética? ¿Su tutor tiene ética? ¿Considera que usted tiene ética? Para una cuarta parte del estudiantado, el programa de posgrado no es ético, hay una mayor proporción de falta de ética en los programas de instituciones públicas y un porcentaje similar expresó que su tutor o tutora no guardan la ética. Además, en varias instituciones privadas sus programas no tienen reconocimiento oficial y también se asumen como universidades sin serlo. Algo que destacó fue que las personas tutoras no acompañan al estudiantado y algunas publican como primeras autoras los trabajos de sus estudiantes. Otros grupos más presentan ponencias en congresos usando los avances de sus estudiantes.

Diez-Martínez (2015) marca una diferencia con las investigaciones que indagaron en la percepción de un sujeto actor acerca de las prácticas deshonestas del otro, porque analizó la deshonestidad académica en alumnado y profesorado en México. Para la autora, la deshonestidad académica es similar al fraude académico, porque se busca un "beneficio académico, económico, afectivo... que [va] en contra de los principios éticos de las instituciones educativas y, en consecuencia, de la sociedad" (Diez-Martínez, 2015, p. 3). La corrupción, agregó, en los sistemas escolares tiene tres niveles. El primero es el macro, integrado y promovido desde las instancias gubernamentales, tales como la malversación y desviación de fondos y la práctica del "clientelismo", la cual consiste en el intercambio de favores y lealtad. El segundo es el institucional, el de la gestión en los organismos educativos, promovido por personal directivo, principalmente. Un tercero, más acotado, es el espacio del aula con las prácticas que realiza el estudiantado (copiar tareas y respuestas en exámenes; copiar y pegar documentos de internet; plagiar textos sin referencias) y el profesorado (opacidad en la asignación de calificaciones; favoritismo; no revisar trabajos; cambiar las formas de evaluación; preguntar temas no vistos 
doi: http://dx.doi.org/10.15359/ree.21-2.12

URL: http://www.una.ac.cr/educare

CORREO: educare@una.cr

en clase). Lo grave es que el estudiantado incorpora las acciones realizadas por otras personas, volviéndolas cotidianas, familiares y necesarias. Las conductas deshonestas impiden generar un espíritu crítico, autónomo y honesto en los jóvenes y las jóvenes y en el profesorado.

Para Navia y Hirsch (2015), la expansión tecnológica ha propiciado que las personas prioricen en los fines sin importar los medios, aunado a la poca oferta de trabajo. Destaca la relación que se mantiene entre los principios éticos que operan en una institución y los que incorpora la persona, de suerte que una institución con principios laxos tendrá personal trabajador con esas nociones e inversamente. De acuerdo con este principio, el actor y la actora está inmerso en circunstancias que limitan sus prácticas, problemática que no es local sino global. En el cuestionario escala que aplicaron a dos muestras de estudiantes de posgrado de dos universidades mexicanas, encontraron que la dimensión ética "Prestar el mejor servicio a la sociedad" tuvo una baja posición, lo que indica que este principio central de la ética profesional fue débil.

Pérez-Castro (2010) realizó una investigación que denominó exploratorio-descriptiva con estudiantes de posgrado de una universidad del sureste mexicano. Sostiene que en la estructura de la ética profesional participan personal directivo, profesorado y estudiantes. Además, la formación prioriza los contenidos disciplinarios y menos los de valores y responsabilidad, lo que provoca un déficit en la formación profesional. En sus resultados encontró una paradoja, porque un porcentaje alto de estudiantes tuvo alta valoración por la responsabilidad, no así por la comunicación, el trabajo en equipo y el compañerismo, elementos necesarios en la ética profesional.

Para Moreno y Romero (2011), la dinámica de evaluación-certificación en los programas de posgrado nacionales ha alterado el ethos de investigación y promovido la aparición de prácticas no adecuadas o vicios éticos (violación a la norma). En ellos participan tanto las autoridades de los programas, como profesorado y estudiantes. Entre estas se encuentra el plagio, la ausencia de paráfrasis, apropiación de marcos conceptuales, pruebas artificiales de hipótesis o supuestos, simulación. Hirsch (2012), Amador-Bautista, Gallegos-Duarte y Arévalo-Guízar (2014) enfatizaron en el plagio académico que va desde páginas de artículos hasta capítulos de tesis para fortalecer otras tesis, porque en los últimos tiempos, el lema es publicar o perecer. Quien no publica no obtiene el premio al mérito, ni certificación en el Sistema Nacional de Investigadores (SNI).

Aluja y Birke (2004) dan un paso importante en el reconocimiento de las prácticas poco éticas en las instituciones educativas de educación superior. Su particularidad radica en que estudian las prácticas realizadas a diario en instituciones de ciencia y formación de maestrías y doctorados y que pueden pasar desapercibidas. Además, los códigos de ética científicos no las han tipificado, por eso denominan "éticamente cuestionables". Son prácticas no 
visibles, incluso en ocasiones pueden justificarse porque atañen a estudiantes, profesorado y autoridades. Dentro de estas están la búsqueda de comisiones dictaminadoras blandas; jurados para exámenes de grado integrados por amistades; fabricación, selección u omisión de datos para confirmar supuestos o hipótesis; omisión de autorías importantes en las investigaciones; autorías injustificadas; evaluación favorable de proyectos de investigación y tesis de personas cercanas; diseñar programas de posgrado sin infraestructura y sin planta académica suficiente y adecuada; alterar cifras para lograr acreditación de un programa de posgrado; concentración de estudiantes en una persona tutora; inflar el curriculum vitae, entre otros.

Montaña (2004), en esta misma línea de prácticas no visibles, menciona la asignación corporativa de estudiantes en un grupo de personas; el sacrificio de la calidad de las tesis y las publicaciones para incrementar la graduación; deficiencia en los criterios de ingreso de estudiantes a un posgrado; ausencia de una línea de investigación original; pertenencia a una cofradía -un pacto por el cual cada persona investigadora de la 'cofradía' incluye como coautoras o coautores de sus trabajos a todos los miembros-. De esta manera, cada quien "multiplica artificialmente su número de publicaciones y transita rápidamente por el camino del tabulador" (p. 252). Pérez-Castro (2014) agrega otras más: someter a dictamen en dos o más revistas el mismo artículo; publicaciones duplicadas; titularidad en los artículos colectivos; armar núcleos académicos artificiales en los programas de posgrado; publicar libros colectivos sin coherencia entre los capítulos; anotar erróneamente las fuentes consultadas. La presión para obtener el doctorado en el tiempo reglamentario puede inducir a disfrazar los datos empíricos; no sistematizar la información de los sujetos entrevistados; exponer como propias ideas vertidas por otros individuos; citar sin atender normas de algún modelo o combinar inapropiadamente varios; defender ante un jurado escritos académicamente débiles; aprobar tesis, artículos o libros con fallas estructurales y de contenido.

Díaz-Barriga (2009) apunta que en México surgieron problemas académicos porque se ha asociado evaluación con calidad y esto como acreditación. Se enfatiza en cumplir con los indicadores de un formato y menos en la dinámica académica de cada programa. La evaluacióncertificación molesta al profesorado, coordinaciones y autoridades, sin embargo, esperarán la certificación. Para cumplir satisfactoriamente adaptarán números, exaltarán fortalezas, ocultarán debilidades, presionarán a personal tutor y estudiantes para agilizar la graduación.

\section{Balance de la bibliografía especializada}

En varias investigaciones antes expuestas, las personas autoras reconocen que los cambios en las políticas educativas y el impacto tecnológico de las últimas décadas han alterado las prácticas en numerosos campos de la vida humana, incluyendo la académica. Esto ha generado prácticas éticamente negativas que empobrecen la formación de la juventud y de futuros y 
doi: http://dx.doi.org/10.15359/ree.21-2.12

URL: http://www.una.ac.cr/educare

CORREO: educare@una.cr

futuras profesionales, porque se educa también con acciones. Estas prácticas las denominan deshonestidad académica, conductas no éticas, vicios éticos, prácticas no éticas, entre otras. Algunas de estas son visibles por la mayoría de integrantes de una comunidad académica. Una que destaca es el fraude en exámenes, tareas, trabajos académicos y publicaciones. La copia o el plagio son prácticas reprobables, porque se atenta directamente contra el deber de toda institución educativa y contra los valores de una formación educativa. Sin embargo, especialmente en el nivel superior se generan otras prácticas deshonestas más sutiles, poco visibles; incluso algunas de ellas justificadas, por ejemplo: búsqueda de jurados laxos para evaluar proyectos artículos y exámenes profesionales; selección de autores y autoras en los artículos; autorías injustificadas; modificación y alteración en las cifras para la certificación de programas; someter un mismo artículo en varias revistas; apoyar a los académicos y las académicas y estudiantes cercanos al círculo, entre otras.

Algunos reportes se centraron en buscar las causas que motivan a realizar alguna práctica deshonesta. Se llega a considerar que responde a la heterogeneidad del estudiantado y a sus condiciones particulares de vida. Para otras personas, son los pocos conocimientos y habilidades académicas para aprobar exámenes o realizar trabajos y artículos. En la mayoría de investigaciones las personas autoras ubicaron el problema en su país o región; no obstante, otras personas enfatizaron que esto no es exclusivo de un país, sino un problema propio de la sociedad contemporánea. Quienes trabajaron el nivel superior apuntaron que los problemas de deshonestidad académica atañen tanto al estudiantado como al profesorado, pero también a los comités dictaminadores, evaluadores y coordinadores. La demanda por una elevada productividad, eficiencia de los procesos, competitividad entre pares y evaluación de procesos y productos crea las condiciones para que las personas adapten y usen las nuevas reglas de evaluación en su beneficio; o bien, que asuman su rol de profesionales y sus actos en investigación y docencia con ética. Veamos qué ocurre con las tesis doctorales analizadas.

\section{Estrategia metodológica}

Para seleccionar las tesis doctorales se visitaron diferentes programas de posgrado en educación. Las tesis se seleccionaron al azar. Algunas se obtuvieron de manera impresa, otras en $C D$ y unas más en red. En total se analizaron 15 tesis de doctorado.

Para el análisis de las tesis fue importante la propuesta de Espino (2015), quien considera que en la elaboración de un documento de posgrado es indispensable que el estudiantado posea las habilidades para la lectura y escritura de textos académicos. No se trata solo de leer numerosos trabajos, sino aquellos que son pertinentes con el objeto de estudio, además leerlos académicamente, con el propósito de escribir claramente las ideas de otros estudios revisados y las propias. 
En el posgrado se exige profundidad para desarrollar el contenido del asunto y la impecable presentación escrita del texto, así como "un abordaje en profundidad de la tarea, donde el producto final implica la lectura de más de una fuente y la elaboración de un trabajo cohesionado que requiere de la integración de la información de los textos consultados... así como de la generación de una estructura propia" (Espino, 2015, p. 961). Lo anterior involucra, necesariamente, procesos de elaboración conceptual para lograr una exposición coherente de la temática. Esta tarea no es sencilla, requiere de una ejercitación diaria, de lectura, análisis de lo leído y manejo de los conceptos de los autores y autoras, así como de los que se adoptarán para la propia investigación. Lo mismo ocurre con la metodología diseñada para la obtención e interpretación de información empírica. No es suficiente con diseñar, probar y aplicar un instrumento de recolección de información, se debe seleccionar aquel que es pertinente con el objeto y el universo de estudio; el cual permitirá obtener la información empírica necesaria para probar los supuestos inicialmente anunciados. Los datos se sistematizan, se construyen categorías, se fundamentan y organizan las ideas. Por último, la redacción del informe final, actividad imposible de evadir.

Gran cantidad de estudiantes puede enfrentarse con numerosas dificultades en este proceso de construcción de la tesis doctoral. Una de ellas es la carencia de tiempo completo para dedicarlo a la investigación. Otra es la poca ejercitación en las habilidades indispensables para la lectura y escritura académicas, así como en la selección y el diseño de instrumentos. Estas situaciones, sin ser justificación, pueden estar vinculadas con las prácticas deshonestas.

La investigación es de carácter exploratorio-descriptiva. Para el análisis de los trabajos se diseñó una escala tipo Likert (Elejabarrieta e Iñiguez, 2008) para valorar por separado 2 dimensiones: contenido y presentación. El contenido exige al estudiantado dominar la lectura especializada con altos niveles de razonamiento, para integrar en un texto las aportaciones de diversas fuentes que le permitan profundizar coherentemente en el tópico a estudiar. La presentación requiere combinar la comprensión lectora con la composición escrita, utilizando procesos cognitivos como la habilidad para examinar, analizar, sintetizar y ser capaz de construir un texto original y coherente, sin errores de ortografía o redacción, que exponga de manera clara y entendible la temática a desarrollar. Dicho instrumento contiene un conjunto de rasgos a los cuales se les asigna un valor, el cual permite examinar con detalle y profundidad los elementos estructurales de las tesis.

Para la dimensión contenido, se consideró primeramente originalidad de la temática y coherencia en la argumentación para sostener las ideas principales de la tesis. Este aspecto se centra en la posibilidad de encontrar nuevas perspectivas de análisis para el objeto de estudio a partir de la consulta de diversas fuentes teóricas, lo cual repercute en la adopción de posturas propias (Arnoux, et. al., 2004). También se valoró la exposición de la problemática de investigación y su coherencia con los objetivos, las preguntas de estudio y la hipótesis o 
doi: http://dx.doi.org/10.15359/ree.21-2.12

URL: http://www.una.ac.cr/educare

CORREO: educare@una.cr

supuestos. En este renglón se recupera lo que señalan especialistas del campo (Ortega-Salas y Sánchez-Hernández, 2009) acerca de la dificultad del estudiantado para integrar en un texto puntos de vista propios, que rebasen lo dicho por los autores y las autoras. En la revisión de las tesis se profundizó en la descripción detallada del proceso metodológico, así como en la pertinencia de los instrumentos de campo y su vinculación con los resultados encontrados. Como parte de este rubro se examinó la presentación de hallazgos y conclusiones, en función de los objetivos señalados para el trabajo. Dichos logros no se alcanzan cuando el estudiantado -aun el que cursa posgrados- al carecer de las habilidades ya mencionadas, se limita a reproducir lo leído en los textos de consulta, sin generar nuevas ideas o reflexiones que contribuyan al campo de conocimiento. Aunado a la falta de comprensión de textos especializados por parte de estudiantes de posgrado (Carlino, 2004), las tesis suelen contener errores de ortografía y redacción por ausencia o falta de revisión de los escritos previos. Este problema se origina en muchas ocasiones, en el escaso tiempo dedicado para la realización de los trabajos: las lecturas deben generar ideas que van madurando con el pensamiento y la reflexión; es difícil la producción de ideas novedosas cuando la urgencia por entregar un texto permea el proceso de construcción.

Para la dimensión presentación, se ahondó en las características del formato: se consideró la claridad en la exposición del tema, la organización del contenido en el índice y en los apartados que lo integran, capítulos y subcapítulos. Una estructura coherente exige claridad en el pensamiento, para una exposición lógica y pertinente en el desarrollo de los diferentes apartados. Asimismo, se valoró la relación entre el título del escrito con el contenido del texto, analizando la manera cómo el título se ve reflejado en el desarrollo del trabajo y en los resultados presentados. En este rubro, se observa la necesidad de que el estudiantado revise de manera continua y consistente investigaciones ya publicadas relacionadas con la temática a estudiar, que se familiaricen con la estructura de los escritos académicos y discutan las diversas formas de organizar la presentación de los contenidos (Espino, 2015). Como parte de esta dimensión, también se revisó a profundidad el estilo, escudriñando en la redacción y estructura de las ideas y los párrafos, situando la coherencia lógica en la exposición de las ideas, en torno a una problemática central que nunca se pierde. Se examinó el uso de la gramática en la expresión escrita, así como la ortografía y puntuación (Arnoux, et al., 2004). Como parte del aparato crítico, se revisaron las citas y referencias al interior del texto y a pie de página, observando las características técnicas y uniformidad en el modelo utilizado. Esta valoración concluye con la contrastación entre las fuentes de información enlistadas en las referencias y las fuentes citadas al interior del texto. A continuación, se muestran los rasgos de cada dimensión (Tabla 1): 
Tabla 1: Rasgos para valorar contenido y presentación

\begin{tabular}{|ll|}
\hline Contenido & Presentación \\
$\square \square$ Originalidad del tema & $\square$ Claridad en el tema \\
$\square$ Argumentación lógica & $\square$ Organización del contenido \\
$\square$ Problemática & $\square$ Índice \\
$\square$ Objetivos & $\square$ Apartados \\
$\square$ Preguntas de estudio & $\square$ Coherencia en exposición de ideas \\
$\square$ Hipótesis/supuestos & $\square$ Uso de la gramática \\
$\square$ Proceso metodológico & $\square$ Ortografía \\
$\square$ Resultados & $\square$ Puntuación \\
$\square$ Hallazgos/conclusiones & $\square$ Citas al interior del texto \\
$\square$ Fuentes de información & $\square$ Referencias/fuente de información \\
\hline
\end{tabular}

Nota: Elaboración propia.

Con el propósito de implementar un instrumento que permitiera evaluar numéricamente los diferentes rasgos de cada dimensión -contenido y presentación-, se diseñó una escala tipo Lickert con 3 niveles de valoración: el más alto -totalmente de acuerdo-al que se le asignó valor de 10 puntos; el intermedio - parcialmente de acuerdo-con valor de 5 puntos y el más bajo -totalmente en desacuerdocon valor de 0 puntos. Una vez determinados los rasgos de cada dimensión y la escala de evaluación, se procedió a la revisión minuciosa de cada trabajo, utilizando la escala tipo Lickert. En la Figura 1 se presentan las puntuaciones otorgadas a las tesis según las características de cada trabajo:

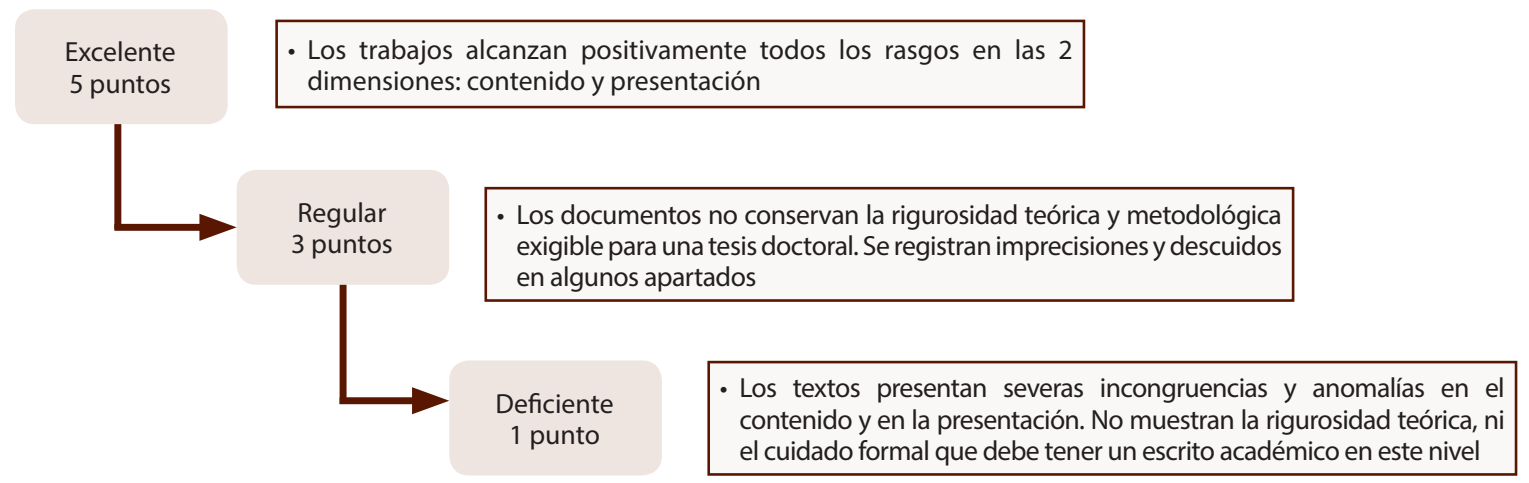

Figura 1: Puntuaciones y valoración de los puntajes

Nota: Elaboración propia. 
doi: http://dx.doi.org/10.15359/ree.21-2.12

URL: http://www.una.ac.cr/educare

CORREO: educare@una.cr

Según se muestra en la Figura 1, las puntuaciones se otorgaron según las características de cada trabajo, asignando la puntuación de 5 a las tesis consideradas excelentes; en el nivel regular se dio puntuación de 3 a aquellas con algunas debilidades teóricas o metodológicas; por último, en el nivel deficiente se otorgó 1 punto por considerar que estos documentos presentan severas deficiencias y no corresponden a un trabajo de posgrado.

\section{Resultados}

A continuación, se presentan los resultados obtenidos de cada trabajo analizado en las dimensiones contenido y presentación. Se obtuvo la sumatoria y se promedió para obtener el puntaje final otorgado a cada tesis.

\section{Contenido:}

Excelente: $5 \quad$ Regular: $3 \quad$ Deficiente: 1

Tabla 2: Resultados del análisis de contenido

\begin{tabular}{|c|c|c|c|c|c|c|c|}
\hline $\begin{array}{l}\text { Número } \\
\text { de tesis }\end{array}$ & $\begin{array}{c}\text { Originalidad } \\
\text { en las ideas y } \\
\text { argumentación }\end{array}$ & $\begin{array}{l}\text { Congruencia entre } \\
\text { título, contenido y } \\
\text { argumentación lógica }\end{array}$ & $\begin{array}{l}\text { Dominio } \\
\text { de estudios } \\
\text { citados }\end{array}$ & $\begin{array}{l}\text { Pertinencia } \\
\text { de estudios } \\
\text { citados }\end{array}$ & $\begin{array}{l}\text { Hallazgos y } \\
\text { conclusiones } \\
\text { relevantes }\end{array}$ & $\begin{array}{l}\text { Puntaje } \\
\text { total }\end{array}$ & Calificación \\
\hline 1 & 5 & 5 & 5 & 5 & 3 & 23 & 9.2 \\
\hline 2 & 3 & 1 & 3 & 3 & 3 & 13 & 5.2 \\
\hline 3 & 3 & 3 & 1 & 3 & 3 & 13 & 5.2 \\
\hline 4 & 3 & 3 & 5 & 5 & 3 & 19 & 7.6 \\
\hline 5 & 1 & 1 & 1 & 1 & 1 & 5 & 2 \\
\hline 6 & 5 & 5 & 5 & 5 & 5 & 25 & 10 \\
\hline 7 & 5 & 3 & 3 & 5 & 5 & 21 & 8.4 \\
\hline 8 & 5 & 5 & 5 & 5 & 5 & 25 & 10 \\
\hline 9 & 3 & 3 & 3 & 3 & 3 & 15 & 6 \\
\hline 10 & 3 & 1 & 3 & 3 & 3 & 13 & 5.2 \\
\hline 11 & 5 & 3 & 3 & 5 & 5 & 21 & 8.4 \\
\hline 12 & 3 & 1 & 3 & 1 & 1 & 9 & 3.6 \\
\hline 13 & 1 & 3 & 1 & 3 & 1 & 9 & 3.6 \\
\hline 14 & 3 & 1 & 5 & 3 & 3 & 15 & 6 \\
\hline 15 & 3 & 3 & 3 & 3 & 3 & 15 & 6 \\
\hline Total & 51 & 41 & 49 & 53 & 47 & 241 & 6.43 \\
\hline
\end{tabular}

Nota: Elaboración propia. 
doi: http://dx.doi.org/10.15359/ree.21-2.12

URL: http://www.una.ac.cr/educare

Como se observa en la Tabla 2, 2 trabajos alcanzaron la máxima puntuación con 25 unidades, 3 obtuvieron 23 y 21 puntos situándose en el nivel excelente; 7 lograron el nivel regular con 19,15 y 13 puntos y 3 consiguieron deficiente con 9 y 5 puntos. Las cifras muestran que, en contenido, $33 \%$ de las tesis analizadas fueron realizadas con el cuidado que corresponde a un trabajo académico comprometido con su quehacer ético y profesional; $46 \%$ fueron trabajos elaborados sin el rigor que exige obtener un grado académico y $20 \%$ no reúne las características mínimas para ostentarse como tesis de posgrado, ni como productos académicos publicables.

\section{Presentación:}

Excelente: $5 \quad$ Regular: $3 \quad$ Deficiente: 1

Tabla 3: Resultados del análisis de presentación

\begin{tabular}{|c|c|c|c|c|c|c|c|}
\hline $\begin{array}{l}\text { Número } \\
\text { de tesis }\end{array}$ & $\begin{array}{l}\text { Estilo: } \\
\text { redacción } \\
\text { de ideas, } \\
\text { estructura } \\
\text { de párrafos }\end{array}$ & $\begin{array}{l}\text { Estructura lógica } \\
\text { del índice: } \\
\text { extensión, } \\
\text { capitulado }\end{array}$ & $\begin{array}{l}\text { Ortografía y } \\
\text { puntuación }\end{array}$ & $\begin{array}{c}\text { Citas y } \\
\text { referencias al } \\
\text { interior del texto, } \\
\text { a pie de página y } \\
\text { testimoniales }\end{array}$ & $\begin{array}{c}\text { Fuentes de } \\
\text { información: } \\
\text { congruencia } \\
\text { de cita de } \\
\text { fuentes con la } \\
\text { bibliografía }\end{array}$ & $\begin{array}{c}\text { Puntaje } \\
\text { total }\end{array}$ & Calificación \\
\hline 1 & 5 & 1 & 5 & 3 & 1 & 15 & 6 \\
\hline 2 & 5 & 1 & 3 & 3 & 3 & 15 & 6 \\
\hline 3 & 3 & 3 & 3 & 1 & 1 & 11 & 4.4 \\
\hline 4 & 5 & 3 & 5 & 5 & 5 & 23 & 9.2 \\
\hline 5 & 3 & 1 & 3 & 1 & 1 & 9 & 3.6 \\
\hline 6 & 5 & 3 & 5 & 5 & 5 & 23 & 9.2 \\
\hline 7 & 5 & 5 & 3 & 3 & 5 & 21 & 8.4 \\
\hline 8 & 5 & 3 & 5 & 3 & 5 & 21 & 8.4 \\
\hline 9 & 5 & 1 & 5 & 3 & 5 & 19 & 7.6 \\
\hline 10 & 5 & 3 & 5 & 5 & 5 & 23 & 9.2 \\
\hline 11 & 5 & 3 & 5 & 5 & 5 & 23 & 9.2 \\
\hline 12 & 1 & 1 & 3 & 1 & 3 & 9 & 3.6 \\
\hline 13 & 5 & 3 & 5 & 3 & 3 & 19 & 7.6 \\
\hline 14 & 5 & 1 & 5 & 3 & 3 & 17 & 6.8 \\
\hline 15 & 3 & 1 & 5 & 3 & 5 & 17 & 6.8 \\
\hline Total & 65 & 33 & 65 & 47 & 55 & 265 & 7.07 \\
\hline
\end{tabular}

Nota: Elaboración propia. 
doi: http://dx.doi.org/10.15359/ree.21-2.12

URL: http://www.una.ac.cr/educare

CORREO: educare@una.cr

Como se observa en la Tabla 3, en presentación 6 trabajos alcanzaron 23 y 21 unidades, que corresponde a excelente; 6 al nivel regular con 19, 17 y 15 puntos y 3 obtuvieron deficiente con 11 y 9 puntos. Puede señalarse que hay tesis como la 5 y 12 que casi en todos los aspectos obtuvieron la mínima puntuación, lo que indica la consistente deficiencia de los trabajos. Las cifras anteriores muestran que, en la presentación, $40 \%$ de los trabajos fueron elaborados con la exigencia requerida para profesionales de la investigación; $40 \%$ son trabajos diseñados con poco cuidado, olvidan el compromiso ético de la profesión y $20 \%$ carece de los rasgos mínimos que deben caracterizar las tesis doctorales. En la concentración de resultados se encontraron los siguientes valores, expuestos en la Tabla 4:

Tabla 4: Concentrado de resultados de las 15 tesis analizadas

\begin{tabular}{cccccc}
\hline No. de tesis & $\begin{array}{c}\text { Dimensión } 1 \\
\text { contenido }\end{array}$ & $\begin{array}{c}\text { Dimensión 2 } \\
\text { presentación }\end{array}$ & $\begin{array}{c}\text { Puntaje } \\
\text { Total }\end{array}$ & $\begin{array}{c}\text { Valor } \\
\text { numérico }\end{array}$ & Nivel \\
\hline 1 & 100 & 100 & 200 & 10 & Excelente \\
5 & 100 & 100 & 200 & 10 & Excelente \\
10 & 95 & 100 & 195 & 9.75 & Excelente \\
14 & 100 & 95 & 195 & 9.75 & Excelente \\
13 & 90 & 95 & 185 & 9.25 & Excelente \\
11 & 90 & 90 & 180 & 9 & Excelente \\
\hline 3 & 90 & 85 & 175 & 8.75 & Regular \\
6 & 90 & 85 & 175 & 8.75 & Regular \\
9 & 95 & 80 & 175 & 8.75 & Regular \\
7 & 100 & 70 & 170 & 8.5 & Regular \\
2 & 85 & 75 & 160 & 8 & Regular \\
\hline 8 & 75 & 50 & 125 & 6.25 & Deficiente \\
15 & 45 & 75 & 120 & 6 & Deficiente \\
4 & 45 & 65 & 110 & 5.5 & Deficiente \\
\hline Promedio & 82.66 & 65 & 5.25 & Deficiente \\
\hline
\end{tabular}

Nota: Elaboración propia. 
De acuerdo con los datos de la Tabla 4, únicamente 6 trabajos obtuvieron las máximas puntuaciones, combinando un contenido relevante con una óptima presentación, lo que justifica la categoría de excelencia. Según se observa en los resultados anteriores, $40 \%$ fueron tesis valoradas en el nivel excelente, lo que significa que, tanto en el contenido como en la presentación, los textos reúnen los rasgos que exige un trabajo académico de posgrado. El segundo grupo en la categoría regular obtuvo 33.3\%, con trabajos que presentan algunas deficiencias en uno o ambas dimensiones, pero pueden considerarse aceptables. El último grupo alcanzó $26.6 \%$ en el rango deficiente, con trabajos de tesis que no reúnen las características de investigaciones doctorales en los contenidos expuestos, ni en la forma de exposición y que, sin embargo, ya fueron aprobados por un comité tutorial en un examen. Los datos anteriores conducen a la necesaria reflexión sobre la ética del personal investigador educativo y el compromiso con su trabajo escrito, así como la manifestación de su ethos que le lleva a orientar sus acciones en cierto sentido.

\section{Discusión}

En la revisión bibliográfica destacaron dos líneas de discusión. Una de ellas se centró en la construcción social del ethos de una disciplina o profesión. En este proceso participan colegas, personal de tutoría y profesorado del programa, entre otras personas. Las prácticas generadas en el grupo, que imperan en la institución y en la disciplina, se aprenden en este espacio. Es producto social, no obstante, el ethos se expresa individualmente, con autonomía y responsabilidad. Este proceso de construcción del ethos responde a lo que Merton (2002), Hamui (2008), Chavoya (2001) y Colina (2011) Ilamaron incorporación de normas, principios y valores del campo disciplinar. Es acoger el oficio de investigar (Sánchez, 2000; Sánchez et al., 2000). La otra línea encontrada indica que las prácticas realizadas en diversos espacios académicos y diferentes niveles educativos tienen un contexto de evaluación que presiona al profesorado de nivel superior: publicar, graduar estudiantes, certificar el programa para tener apoyo financiero y reconocimiento nacional o internacional (Chavoya, 2001). Estas circunstancias son terreno para el cultivo de prácticas no éticas o vicios éticos o prácticas deshonestas, visibles (copiar y pegar párrafos o textos completos, hasta el plagio de libros) (Amador-Bautista et al., 2014; Díaz et al., 2010; Flores-Pérez, 2004; Hirsch, 2012; Moreno y Romero, 2011; Navia y Hirsch, 2015; Pérez-Castro, 2010) y no visibles (adaptación de núcleos académicos, graduaciones aceleradas, simulación académica, maquillaje de estadísticas, tutoría a numerosos estudiantes) (Aluja y Birke, 2004; Montaña, 2004; Pérez-Castro, 2014).

Independientemente de las circunstancias que envuelven estas decisiones, es necesario resaltar que no todos los individuos obedecen a las fuerzas externas, ni imitan las prácticas de otros, porque su acción ética implica autonomía y juicio crítico (Yurén, 2007) elementos imprescindibles en la honestidad académica y en la ética profesional. Una práctica invisible porque poco se menciona en las investigaciones es la que se refiere a la elaboración de tesis 
doi: http://dx.doi.org/10.15359/ree.21-2.12

URL: http://www.una.ac.cr/educare

CORREO: educare@una.cr

de doctorado, la cual sintetiza la formación del estudiantado de un ciclo educativo. Se espera que esta sea una investigación original, cuidada en su contenido, con una metodología pertinente con el objeto, con resultados, con un sólido aparato crítico y con rigor metodológico en la sistematización e interpretación de datos. La tesis, se ha mencionado, es la síntesis de la formación, porque puede representar para el estudiantado el inicio de una línea que cultivará durante varios años, o solo un requisito para presentar un examen y olvidarla. No obstante, tanto en uno como en otro caso, la persona egresada tiene el deber de hacer bien el trabajo, apegarse a los lineamientos conceptuales, instrumentales y morales de la disciplina y de la investigación. Si no hay cuidado en ello, no se está cumpliendo éticamente, sino incurriendo en una práctica que se aleja de este deber. No hay ethos profesional incorporado en el estudiantado, no se incorporaron las normas establecidas en un campo disciplinar (Chavoya, 2001; Hamui, 2008; Merton, 2002; Sánchez, 2014).

La investigación educativa es una profesión que se cultiva día tras día mediante numerosos actos ejecutados responsablemente. Cuando no ocurre eso, se cae en prácticas que van en detrimento del ejercicio profesional. Se hacen por obligación, sin que la persona esté convencida de su trabajo ni de la importancia de realizar los deberes de su profesión. En la revisión de las tesis doctorales, se encontró que una tercera parte de los informes fueron hechos con cuidado, respetando los requisitos que exige una tesis de posgrado, pero en otros hicieron solo lo indispensable, solo aquello que les fue solicitado por la persona tutora, sin mayor esfuerzo. Otros más no respetaron los lineamientos de forma requeridos en todo trabajo de investigación, aunado a numerosos descuidos en el contenido y presentación. En estos casos se presentan prácticas no éticas. Se hace lo que se solicita, incluso ni eso, pero se autorizó la tesis. Entonces, ¿quién es la persona responsable? Sin duda, en primer lugar, el estudiante o la estudiante, de quienes depende la responsabilidad y la autonomía como futuras personas doctoras e investigadoras. En segundo lugar, están el tutor y la tutora y el comité, quienes no acompañaron al estudiantado en su proceso de formación.

Es necesario reconocer, como apuntan Moreno y Romero (2011), que en cada programa se incorporan diversidad de estudiantes, algunos grupos ya con trayectoria en investigación y deseos de convertirse en investigadores o investigadoras profesionales, como otros que tienen serias dificultades para el trabajo académico. Habrá estudiantado autónomo y otro que necesitan dirección. Cada cual tiene una persona tutora y un comité, cuya tarea consiste en conducir y valorar el proceso de formación. Si el estudiantado no cumple, ni su tutor o tutora, ni su comité, se está ante un conflicto ético que se reflejará en el documento final, en parte por las circunstancias creadas por un contexto de producción y evaluación. La tesis doctoral se elabora a lo largo de su estancia en el programa (Sánchez y Jasso, 2002; Sánchez y Santamaría, 2000). Es producto de los seminarios que el estudiantado cursa, de la ejercitación académica en espacios no áulicos con sus compañeros, compañeras y profesorado. En el programa discute su tema y reconoce que 
debe acotarlo hasta convertirlo en un objeto de conocimiento, de las fuentes consultadas, de las técnicas para obtener y sistematizar información empírica de manera novedosa e interesante, etc. En el documento final se expresa la formación y el ethos de cada estudiante.

La investigación educativa es un oficio similar al trabajo. Ejercerla con ética debe ser un principio inherenteen la trayectoria personaly profesional delosinvestigadoresylasinvestigadoras. A partir de los datos encontrados en la investigación de campo, se observó que los principios éticos no siempre están presentes en los productos de la investigación, lo que nos lleva a suponer que otros factores, más allá de la búsqueda del conocimiento, permean el quehacer investigativo.

\section{Conclusiones}

El ethos de una profesión significa incorporación de las reglas teóricas y metodológicas de una disciplina, además de las normas y valores necesarios en un profesional. Es saber hacer lo que la profesión demanda, pero hacerlo apegado a los valores disciplinarios. En el caso de la investigación en general y la educativa en especial, el ethos consiste en saber investigar y apegarse a las normas establecidas por la comunidad de personal investigador, por ejemplo, citar correctamente todas las autorías consultadas, no difundir como propias las ideas de otras personas. Algo más, hacer un buen trabajo en estudiantes de posgrado implica elaborar una tesis apegada a las normas de la investigación, lo cual demanda dedicación, compromiso, revisión permanente de lo que se escribe, todo como un acto ético porque debe hacerse responsable y autónomamente (Yurén, 2007).

En los programas doctorales en investigación educativa se prepara a los futuros investigadores y a las futuras investigadoras. Para esto es indispensable que se ejerciten constantemente en la lectura y en la elaboración de reportes, que escriban ensayos y ponencias para exponerlas en congresos o seminarios; además, que lean y analicen trabajos de sus colegas. Tienen que comunicarse periódicamente con su tutor o tutora y con los sujetos integrantes de su comité tutoral. La interacción entre pares y profesorado es indispensable para ir logrando la identidad con la institución, con un grupo y con la disciplina (Hamui, 2008). La incorporación de un ethos es producto de una construcción social, de la interacción diaria que se entabla en los diversos espacios de un programa, tanto con integrantes de su comité tutorial como con otras personas del profesorado y colegas. En este proceso de formación se prepara en el conocimiento y uso de los conceptos y métodos, como también en las normas técnicas y morales de una disciplina o profesión (Bolívar, 2005; Merton, 2002; Moreno, 2013; Sánchez, 2000; Sánchez et al., 2000).

Sin embargo, señala Chavoya (2001), otra dimensión que interviene en la formación de personal de investigación es el contexto que rodea los programas de posgrado. En el siglo XXI, el proceso de evaluación y certificación de instituciones, programas, personal académico, proyectos de investigación, revistas, etc., ha llevado a que un sector de la planta académica se concentre en publicar y graduar y otro tanto le interesa cumplir con sus tareas académicas. Por 
doi: http://dx.doi.org/10.15359/ree.21-2.12

URL: http://www.una.ac.cr/educare

CORREO: educare@una.cr

tanto, la tutoría se interpreta desde diferentes ángulos. El estudiantado, de igual forma, tiene diversos motivos para estudiar un doctorado, como lo reporta Moreno (2013), demuestran distinto compromiso hacia las tareas académicas y con su formación. Habrá quienes se dediquen completamente a cumplir con las actividades que demanda el programa y quienes harán lo mínimo que les permita permanecer en el programa. La calidad de la tesis de grado es prueba de esto. Los programas de posgrado están inmersos en este contexto histórico.

Con base en la revisión de las 15 tesis doctorales en investigación educativa, solo 6 trabajos mostraron originalidad en el tratamiento académico, tuvieron una argumentación lógica, lograron la construcción de un problema de conocimiento, con precisión en los objetivos, los supuestos de investigación fundamentados y las conclusiones claramente expuestas. Además, la argumentación de cada idea la apoyó en fuentes documentales. En resumen, 40\% fueron tesis valoradas en el nivel excelente. Se trató de documentos con los rasgos que exige un trabajo académico de posgrado. Se puede sostener que se trata de estudiantado comprometido con su quehacer y con la profesión. El segundo grupo, equivalente al 33.3\%, se integró con trabajos con deficiencias, pero pueden considerarse aceptables. Por último, el $26.6 \%$ de los documentos fueron deficientes, porque no poseen los elementos indispensables para una investigación de doctorado, tanto por sus contenidos trabajados, como por la forma de exposición. Resalta que hayan sido avaladas positivamente por un tutor o tutora, así como por el comité tutorial en un examen de grado. Lo antes dicho permite preguntarse, con base en Moreno (2013), acerca del ethos del personal investigador educativo, plasmado en su trabajo escrito, así como el del profesorado que participó en la evaluación de ese documento.

Estos resultados demuestran la existencia de tesis de distinta calidad académica, por tanto, también se presenta un estudiantado diferenciado y una variedad de prácticas educativas. Solo un porcentaje de las tesis demostró un buen y responsable trabajo, expresó compromiso con su tarea y un apego al ethos de la investigación educativa (Chavoya, 2001; Hamui, 2008; Merton, 2002; Sánchez, 2014). Otro sector se alejó de este ideal, específicamente el $26.6 \%$ cuyos documentos fueron deficientes, situación que indica que los programas no son independientes del contexto de evaluación, sino que este se está imponiendo y está afectando las prácticas educativas. Montaña (2004) menciona que el sacrificio de la calidad de las tesis para incrementar la graduación o eficiencia terminal va acompañado con criterios limitados para el ingreso del estudiantado a un posgrado. Ingreso y egreso están relacionados.

Lo importante para la juventud y para quienes integran el comité tutoral es graduar, porque se debe cumplir con cuotas de trabajo. La permisividad en el otorgamiento de votos aprobatorios y de grados, así como la escasa dedicación al trabajo de tesis, demuestran que las prácticas éticamente cuestionables que mencionan Aluja y Birke (2004), o prácticas invisibles, han llegado a estos programas. Son prácticas que se toleran, no se reconocen, incluso pueden responder a acuerdos entre el profesorado, funcionarios y funcionarias, con el objetivo de acelerar la graduación. 
Sin embargo, independientemente de este contexto difícil para el trabajo académico y para la ética de la investigación educativa, se debe reconocer que estas políticas no desaparecerán en el corto plazo. Esto hace necesario buscar posibles salidas en lo interno y particular de los programas. Una opción es consolidar los cuerpos académicos, formados solo con doctores y doctoras con una línea de investigación, reconocidos por la seriedad de su trabajo y su honestidad como personal académico. Su aceptación no es solo por su antigüedad sino por su trabajo constante, responsable y honesto. La selección del estudiantado debe ser estricta, de manera que ingresen quienes porten las habilidades indispensables para las tareas académicas y de investigación. Los comités tutoriales deberán dar ese acompañamiento a los futuros doctores y doctoras, mediante la lectura de los avances y en reuniones sistemáticas y periódicas. Como apuntan Aluja y Birke (2004, p. 137), "pugnar porque a nivel individual e institucional, existan mecanismos de autoevaluación permanente".

La otra propuesta obedece a las interrogantes que se acumularon en la elaboración de este artículo. Guardan relación con las prácticas que personal funcionario y académico despliegan en los programas, para acreditar las evaluaciones personales a sus proyectos de investigación. Se busca la evaluación positiva solo por los recursos económicos que proporciona, o se espera algo más, por ejemplo, el reconocimiento de sus individuos cercanos, el prestigio como investigador o investigadora o el prestigio de un programa, entre otros. Quienes han cometido una falta no ética y que han sido descubiertos, ¿qué argumentos proporcionan en su defensa? Interrogantes que requerirán de la inmersión en los espacios académicos para observar y registrar, aunado a la realización de entrevistas con las personas académicas, tareas pendientes en esta línea de investigación.

\section{Referencias}

Aluja, M. y Birke, A. (2004). Panorama general sobre los principios éticos aplicables a la investigación científica y la educación superior. En M. Aluja y A. Birke (Coords.), El papel de la ética en la investigación científica y la educación superior (pp.87-144). México: Academia Mexicana de las Ciencias.

Amador-Bautista, R., Gallegos-Duarte, L. y Arévalo-Guízar, G. (2014). Desafíos éticos del sistema de evaluación de la productividad académica y la difusión de la investigación. En A. Hirsch y R. López (Coords.), Ética profesional en educación superior. Finalidades, estrategias y desafíos de la formación (pp. 101-126). México: Universidad Autónoma de Sinaloa. Recuperado de https://www.researchgate.net/profile/Manuel Marti Vilar/publication/265599815 Responsabilidad Social y etica en Educacion Superior Marcos y Experiencias/ links/5480750d0cf2ccc7f8bd0573/Responsabilidad-Social-y-etica-en-EducacionSuperior-Marcos-y-Experiencias.pdf 
doi: http://dx.doi.org/10.15359/ree.21-2.12

URL: http://www.una.ac.cr/educare

CORREO: educare@una.cr

Arnoux, E., Borsinger, A., Carlino, P., Di Stefano, M., Pereira, C. y Silvestre, A. (2004). La intervención pedagógica en el proceso de escritura de tesis de posgrado. Revista de la Maestría en Salud Pública, 2(3), 1-16. Recuperado de http://www.aacademica.org/paula.carlino/169.pdf.

Bolívar, A. (2005). El lugar de la ética profesional en la formación universitaria. Revista Mexicana de Investigación Educativa, 10(24), 92-123. Recuperado de http://www.redalyc.org/ articulo.oa?id=14002406

Carlino, P. (2004). El proceso de escritura académica: Cuatro dificultades de la enseñanza universitaria. Educere, 8(26), 321-327. Recuperado de http://www.redalyc.org/articulo. oa?id=35602605

Castillo-Méndez, A. y Garibay-Pardo, L. (2004). Ética en la investigación científica y la educación superior: Perspectiva de una estudiante de licenciatura. En M. Aluja y A. Birke (Coords.), El papel de la ética en la investigación científica y la educación superior (pp. 181-202). México: FCE/Academia Mexicana de las Ciencias.

Chavoya, M. L. (2001). Organización del trabajo y culturas académicas. Estudio de dos grupos de investigadores de la Universidad de Guadalajara. Revista Mexicana de Investigación Educativa, 6(11), 79-93. Recuperado de http://www.redalyc.org/pdf/140/14001106.pdf

Colina, A. (2011). La ética profesional y el narcisismo benigno en la formación de la identidad del investigador en educación en México. Revista lberoamericana de Educación Superior, 2(4), 135-148. Recuperado de http://ries.universia.net/index.php/ries/article/view/110

Díaz, A. J., González, F. D. y Carmona L. E. (2010). Relación del fraude académico con situaciones personales que enfrentan los estudiantes en la Facultad de Odontología de la Universidad de Cartagena (Colombia). Salud Uninorte, 26(1), 85-97. Recuperado de http://www.scielo. org.co/pdf/sun/v26n1/v26n1a10

Díaz-Barriga, A. (2009). Criterios de evaluación externa de los posgrados en México. Un sistema de acreditación que desconoce su pertinencia social. En T. Pacheco y Á. Díaz-Barriga (Coords.), El posgrado en educación en México ( $2^{\mathrm{a}}$ ed., pp. 45- 87). México: UNAM. Recuperado de https://issuu.com/educomplexus/docs/el-posgrado-en-educacion-en-mexico

Diez-Martínez, E. (2015). Deshonestidad académica en alumnos y profesores. Su contribución en la desvinculación moral y corrupción social. Sinéctica, 44, 1-17. Recuperado de https:// sinectica.iteso.mx/index.php/SINECTICA/article/view/161/154

Elejabarrieta, F. J. e lñiguez, L. (2008). Construcción de escalas de actitud, tipo Thurstone y Likert. Revista La Sociología en sus escenarios, 17, 1-47. Recuperado de http://aprendeenlinea. udea.edu.co/revistas/index.php/ceo/article/viewFile/6820/6246 
Espino, S. (2015). La enseñanza de estrategias de escritura y comunicación de textos científicos y académicos a estudiantes de posgrado. Revista Mexicana de Investigación Educativa, 20(26), 959-976. Recuperado de http://comie.org.mx/v1/revista/visualizador.php?articulo=ART66 011\&criterio=http://www.comie.org.mx/documentos/rmie/v20/n066/pdf/66011.pdf

Flores-Pérez, F. I. (2004). El papel de la ética en la investigación científica y la educación superior: Opinión de 189 estudiantes de posgrado. En M. Aluja y A. Birke (Coords.), El papel de la ética en la investigación científica y la educación superior (pp. 203-218). México: FCE/Academia Mexicana de las Ciencias.

Hamui, M. (2008). La identidad en la conformación del ethos: El caso de un grupo de científicos de investigación sobre relaciones internacionales de un instituto de educación superior. Estudios Sociológicos, 26(76), 87-118. Recuperado de http://www.redalyc.org/articulo. oa?id=59826104

Hirsch, A. (2003). Elementos significativos de la ética profesional. Reencuentro, 38, 8-15. http:// www.redalyc.org/pdf/340/34003802.pdf

Hirsch, A. (2012). Consideraciones de los coordinadores de posgrado de la Universidad Nacional Autónoma de México respecto a la ética profesional. En A. Hirsch y R. López (Coords.), Ética profesional en la docencia y la investigación (pp. 91-112). México: UABC, UAT, Universidad Michoacana de San Nicolás de Hidalgo, UPAEP, Ediciones del Lirio.

Merton, R. K. (2002). Teoría y estructuras sociales (4ª ed.). México: FCE.

Montaña, C. (2004). El papel del profesor y director de tesis en la transmisión de valores éticos. En M. Aluja y A. Birke (Coords.), El papel de la ética en la investigación científica y la educación superior (pp. 245-260). México: FCE/Academia Mexicana de las Ciencias.

Moreno, M. G. (2013). Apoyo familiar, compromiso personal y disposición a las rupturas. Una historia de formación doctoral. En C. Barrón y G. A. Valenzuela (Coords.), El posgrado. Programas y prácticas (pp. 133-173). México: IISUE-UNAM. Recuperado de http://www. acuedi.org/ddata/11347.pdf

Moreno, M.G.y Romero, M. A. (2011). Ética, investigación educativa y formación de investigadores: Entre la norma y el proyecto de vida. Revista lberoamericana sobre Calidad, Eficacia y Cambio en Educación, 9(2), 80-96. http://www.redalyc.org/pdf/551/55119127006.pdf

Navia, C. y Hirsch, A. (2015). Ética profesional en estudiantes de posgrado en dos universidades mexicanas. Revista Electrónica de Investigación Educativa, 17(1), 100-115. Recuperado de http://www.scielo.org.mx/pdf/redie/v17n1/v17n1a7.pdf 
doi: http://dx.doi.org/10.15359/ree.21-2.12

URL: http://www.una.ac.cr/educare

CORREO: educare@una.cr

Ortega-Salas, M. y Sánchez-Hernández, S. (2009). Estrategias de enseñanza para elaboración de textos escritos a nivel universitario. En Memorias del $X$ Congreso Nacional de Investigación Educativa (pp. 1-11). Recuperado de http://www.comie.org.mx/congreso/ memoriaelectronica/v10/pdf/area tematica 05/ponencias/0231-F.pdf

Pérez-Castro, J. (2010). Elementos para la reflexión en torno a la ética profesional de los estudiantes de posgrado. Revista Electrónica de Investigación Educativa, Número Especial, 1-17. Recuperado de http://redie.uabc.mx/redie/article/view/253

Pérez-Castro, J. (2014). Dimensiones y problemas éticos en la formación de investigadores. En A. Hirsch y R. López (Coords.), Ética profesional en educación superior. Finalidades, estrategias y desafíos de la formación (pp. 127-154). México: UAS, Universidad de Valencia, UABC, Universidad Católica de Valencia, UAT, Universidad Antonio Nariño, UPAEP, Universidad Luis Amigó, Universidad de Antofagasta, ISCEEM. Recuperado de file:///Users/ FAMPENABADCAMACHO/Downloads/E\%CC\%81tica\%20profesional\%20en\%20la\%20 Educacio\%CC\%81n\%20superior\%20(1).pdf

Sánchez, R. (2000). Referentes teórico-metodológicos. En R. Sánchez y M. Arredondo (Coords.), Posgrado de ciencias sociales y humanidades. Vida académica y eficiencia terminal (pp. 1953). México: CESU-UNAM.

Sánchez, R. (2014). Enseñar a investigar. Una didáctica nueva de la investigación en ciencias sociales y humanas. México: IISUE-UNAM.

Sánchez, R. y Jasso, E. (2002). El programa de maestría y doctorado en ciencias. Neurobiología (Cuadernos del CESU, 38). México: UNAM-CESU.

Sánchez, R. y Santamaría, M. P. (2000). El proceso y las prácticas de tutoría. En R. Sánchez y M. Arredondo (Coords.), Posgrado de ciencias sociales y humanidades. Vida académica y eficiencia terminal (pp. 109- 135). México: CESU-UNAM.

Serna, A., Roa, R., Martínez, A. O. y Aguirre, A. (2014). Competencias y rasgos de ética profesional y valores en la formación de docentes en posgrado. En A. Hirsch y R. López (Coords.), Ética profesional en educación superior. Finalidades, estrategias y desafíos de la formación (pp. 233-259). México: UAS, Universidad de Valencia, UABC, Universidad Católica de Valencia, UAT, Universidad Antonio Nariño, UPAEP, Universidad Luis Amigó, Universidad de Antofagasta, ISCEEM Recuperado de http://roderic.uv.es/ bitstream/handle/10550/41252/\%C3\%89tica\%20profesional\%20en\%20la\%20 Educaci\%C3\%B3n\%20superior.pdf?sequence=1

Sureda, J. y Comas, R. (2009). Prácticas académicas deshonestas en el desarrollo de exámenes entre el alumnado universitario español. Estudios sobre Educación, 17, 103-122. Recuperado de http://dadun.unav.edu/handle/10171/9852 
doi: http://dx.doi.org/10.15359/ree.21-2.12

URL: http://www.una.ac.cr/educare

Sureda, J., Comas, R. y Morey, M. (2009). Las causas del plagio académico entre el alumnado universitario según el profesorado. Revista Iberoamericana de Educación 50, 197-220. Recuperado de www.rieoei.org/rie50a10.pdf

Yurén, M. T. (2007). Ética, derechos y responsabilidad social. En A. Arredondo y J. Góngora, Educación, ética y responsabilidad ciudadana de los docentes (pp.47-56). México: IFE. 